\title{
Estrategias para aumentar el compromiso organizacional en el área de ventas de una empresa de la industria cosmética y cuidado personal
}

\section{Strategies to increase organizational engagement in the sales area of a company in the cosmetics and personal care industry}

López Bravo Noli Jhasmeth

ORCID: https://orcid.org/0000-0002-9311-7448

Dondero Cassano Pietro Pablo Guissepi

ORCID: https://orcid.org/0000-0003-3117-0136

Universidad Norbert Wiener, Perú

Autor para correspondencia: nolilopez43@gmail.com; pietrodondero@yahoo.com

Fecha de recepción: 13 de julio del 2019 - Fecha de aceptación: 25 de octubre del 2019

\section{Resumen}

La presente investigación desarrollada tiene como objetivo proponer estrategias para mejorar el compromiso organizacional del área de ventas de una empresa de la industria cosmética y cuidado personal. El estudio se trabajó mediante el sintagma holístico, enfoque mixto, de tipo proyectiva, nivel comprensivo, método inductivo y deductivo, para la recopilación de datos se utilizó un cuestionario correspondiente a 18 ítems, la población estuvo constituida por 15 asesores de ventas, mientras que las entrevistas se aplicaron a los tres gerentes. El procesamiento y análisis de la información cuantitativo se realizó con el apoyo del diagrama de Pareto, software Spss.24 y Atlas ti para la información cualitativa. El compromiso organizacional se logró medir a través de tres componentes: compromiso afectivo, de continuidad y normativo, teniendo como resultados por parte del personal la falta de involucramiento, falta de motivación y la rotación continua, es por ello que se planteó el Modelo IML que implica actividades de involucramiento, motivación y una línea de carrera para lograr elevar el compromiso organizacional que es importante el logro de los objetivos organizacionales.

Palabras claves: compromiso organizacional; involucramiento; compromiso normativo; compromiso de continuidad; compromiso normativo

\begin{abstract}
The present research developed aims to propose strategies to improve the organizational commitment of the sales area of a company in the cosmetics and personal care industry. The study was worked through holistic term, mixed approach, projective type, comprehensive level, inductive and deductive method, for the collection of data a questionnaire corresponding to 18 items was used, the population consisted of 15 sales advisors, while the interviews were applied to the three managers. The processing and analysis of quantitative information was carried out with the support of the Pareto diagram, Spss.24 software and Atlas.ti for the qualitative. Organizational commitment was measured through three components: affective, continuity and normative commitment, resulting in lack of involvement, lack of motivation and continuous
\end{abstract}


rotation, that is why the IML Model involving engagement activities was raised, motivation and a career line to elevate organizational engagement that is important the achievement of the organizational objectives.

Key words: organizational commitment; involvement; regulatory commitment; commitment to continuity; regulatory commitment

\section{Introducción}

En la actualidad, se reconoce que las personas se han convertido en el capital importante dentro de las empresas, es por ello que las organizaciones están trabajando cada día en satisfacer todas sus necesidades, con la finalidad que las personas se comprometan y trabajen mejor, sin embargo, aún existen empresas que no llegan a satisfacer todas las necesidades, piensan que con renumerarles bien el personal está comprometido, lamentablemente están equivocados.

Aon Corporación empresa consultora de gestión y recursos humanos indicó que el compromiso de los trabajadores aumenta de manera razonable, debido a que están trabajando cada día en mantenerlos alineados e impulsando al cumplimiento de las metas trazadas, un factor muy importante que logró aumentar el compromiso, es la remuneración y reconocimiento, existen tres variables distintas que ayudan a medir el nivel de compromiso del trabajador: La primera corresponde al indicador "hablar" mide el nivel con que el colaborador defiende a la empresa, evidenciándose el incrementó de $68 \%$ a $70 \%$ en el último año, la segunda corresponde al "permanecer" con un resultado de $61 \%$, mide la permanencia de los trabajadores en empresa, y por último "contribuir" que obtuvo un $64 \%$, referida al desenvolvimiento del trabajador en la organización (Aon, 2018).

Según la consultora Deloitte, indica que para el $87 \%$ de empresas, su principal preocupación es la falta de compromiso por parte de los trabajadores, debido a que en la actualidad los nuevos trabajadores (Millennials), quieren desenvolverse desarrollando nuevas formas de trabajar, en el cual las empresas no están reaccionando ante este caso. En este nuevo mundo laboral, las organizaciones deben trabajar innovando cada vez más respecto a cómo gestionar a sus talentos (Gestión, 2015).

Como problema general tenemos la falta de compromiso organizacional por parte de los trabajadores en el área de ventas dentro de una empresa de la industria cosmética y cuidado personal, lo cual está generando disconformidad y preocupación debido al incumplimiento de funciones por parte de las asesoras de venta, falta de trabajo en equipo, problemas de atención y amabilidad con el cliente, falta de identificación con su puesto de trabajo, mala coordinación de horarios, clima laboral inadecuado y falta de motivación y supervisión por parte de la empresa, de tal forma, genera una rotación en el personal de ventas.

Crespo (2016) en su investigación sobre el clima y compromiso organizacional obtuvo como resultados que los trabajadores se sienten comprometidos con dicha empresa, cabe resaltar que el compromiso afectivo es el más relevante, en el cual quiere decir que el trabajador está muy involucrado de una manera emocional con la empresa. Por otro lado Loza (2016) observó un $73 \%$ de encuestados que su compromiso organizacional es suficiente, puesto que los 
trabajadores han constituido lazos emocionales, es decir, la empresa cuenta con trabajadores comprometidos que demuestran su mejor esfuerzo en el trabajo.

Entonces decimos que el compromiso organizacional consiste en el nivel de esfuerzo que tiene el colaborador, a la vez se consideran como pieza clave para llegar a cumplir con los objetivos, logrando tener aprecio hacia la empresa, esto a través de su empeño que ofrece día a día (Alles, 2007).

Fuentes (2018) determinó en su estudio que el compromiso organizacional es débil con $48.3 \%$, moderado con $46.7 \%$ y fuerte con 5\%. Asimismo, Paredes (2017) en su estudio obtuvo como resultado que el compromiso organizacional de los trabajadores de la empresa es adecuado con $56 \%$, y desfavorable con $35.71 \%$

Elevar el compromiso organizacional de los trabajadores en el área de ventas es muy importante, debido a que si se sienten muy involucrados con la empresa mejor será el cumplimiento de los objetivos (Amoros, 2007) y esto conlleva que aumente la rentabilidad de la empresa a través de las buenas ventas que realicen, es por ello que la organización debe satisfacer todas las necesidades que el trabajador requiere, impulsándolos mediante motivaciones y así se logre un buen desempeño laboral e identificándose con la empresa. Tal como menciona Maslow en su pirámide representada mediante un orden jerárquico de necesidades, pues está constituido por cinco niveles, en el cual se encuentran de una manera ordenada y ascendente según su relevancia para su pertenencia y capacidad de motivación (Chiavenato, 2014).

En esta investigación se revisó también la teoría de compromiso de Becker, la cual nos enseña una variedad de tipos de compromiso los cuales son compromiso: ante la organización, ante las jefaturas, compromiso frente al supervisor, frente a los equipos de trabajo, y frente a las funciones a realizar, de tal manera, que cada uno de los distintos tipos de compromiso mencionados tienen su propia mecánica de influir en el desempeño de cada uno de los colaboradores dentro de la empresa, asimismo influye en la conducta organizacional. Todo esto en absoluto vienen hacer mecanismos de la acción laboral y organizacional de los trabajadores que se enlazan con el mejoramiento y desarrollo dentro de la organización (Becker ,1992). Y también se revisó la teoría X y Y de Douglas McGregor, lo cual permite percibir como desarrollan sus actividades las asesoras de ventas, se conocerá quienes trabajan bajo el perfil de la teoría X y quienes trabajan bajo el perfil de la teoría Y. (Mercado,2002).

El compromiso organizacional consiste en la identificación de un colaborador con la organización, respecto a los objetivos, logrando ser parte fundamental e importante de la organización en donde pertenecen (Robbins \& Coulter, 2014). Se consideró para esta investigación las siguientes subcategorías con sus respectivas dimensiones:

Compromiso afectivo: consiste en un apego psicológico emocional, la voluntad, el gusto y la filiación de los trabajadores con cada una de las metas de la organización (Fields, 2013).

Compromiso de continuidad: se basa con los beneficios económicos que tiene un colaborador, dentro de una organización, el cual es consiente lo que perdería si llegara a renunciar (Meyer \& Allen, 1991). 
Compromiso normativo: está relacionado cuando un trabajador se siente en la obligación de pertenecer a la organización por gratitud, por estima, también por el tiempo que labora ya que se sienten con el deber de continuar en la empresa (Meyer \& Allen, 1991).

Se tiene como objetivo general proponer estrategias para mejorar el compromiso organizacional del área de ventas de una empresa de la industria cosmética y cuidado personal y los objetivos específicos son analizar el compromiso organizacional del área de ventas de una empresa de la industria cosmética y cuidado personal y explicar los factores de mayor incidencia en el compromiso organizacional del área de ventas de una empresa de la industria cosmética y cuidado personal.

\section{Métodos}

Se realizó mediante el sintagma holístico que consiste en la unión y diversificación de información (Hurtado,2000), enfoque mixto (cualitativos y cuantitativos) lo cual se realizaron encuestas y entrevistas, (Hernández, Fernández, \& Baptista,2010), de tipo proyectiva que está relacionada en buscar, y crear ideas, y así proponer un plan de soluciones el problema identificado (Hurtado,2010), método inductivo que se refiere a fundamentarse en enunciados sencillos como los detalles de los resultados a través de la observación y experiencia (Cegarra, 2012), y nivel comprensivo (Romeu, 1999).

\section{Ficha técnica del cuestionario utilizado}

Nombre: Cuestionario del compromiso organizacional

Autores: Meyer y Allen

Procedencia: EE. UU

Objetivo: Analizar el compromiso organizacional que presenta las asesoras de venta

Estructuración: consta de tres componentes (compromiso afectivo, compromiso normativo, compromiso de continuidad) con un total de 18 ítems.

Escala tipo: Likert

Duración: 10 minutos

Para la validez de los instrumentos se utilizó el juicio de expertos y para la confiabilidad del instrumento se utilizó el Coeficiente Alfa de Cron Bach. En cuanto a las unidades informantes estuvo constituida por 15 asesoras de ventas, Además, contamos con tres gerentes para las entrevistas, la gerente general, la gerente de marketing y la gerente de operaciones. Respecto al análisis cuantitativo se usó diagrama Pareto, software Spss.24 y los datos cualitativos se realizó la triangulación aplicando el programa Atlas.ti.

\section{Resultados}

\section{Resultados cuantitativos}

De acuerdo a la subcategoría compromiso afectivo los resultados de las encuestas realizadas a los asesores de ventas, se determinó que el 33.33\% están de acuerdo que la empresa 
tiene un significado para ellos, un $33.33 \%$ mencionaron sentirse que forman parte de una familia en la empresa, pero un $26.67 \%$ indicaron que no sienten suyos los problemas de la organización. Un $40 \%$ mencionó que les gusta lo que hacen en el trabajo de la misma manera un $40 \%$ mencionaron tener una fuerte sensación de pertenecer en la organización. Un $33.33 \%$ disfruta hablar de la empresa y por último un $33.33 \%$ estuvieron en desacuerdo en seguir laborando de manera permanentemente en la organización.

En cuanto a los resultados de la subcategoría compromiso de continuidad, se determinó que el $40 \%$ de los encuestados están de acuerdo con el sueldo actual y las oportunidades ya que son mejores que otras empresas del mismo rubro. Pero a la misma vez un $40 \%$ mencionaron que el trabajo no cumple todas las expectativas para seguir laborando, además un $46.67 \%$ dijeron que sería difícil conseguir trabajo como el que tiene, de tal forma que un $40 \%$ mencionó que sería difícil dejar la organización y a la vez un $46.67 \%$ opinó que ninguna cosa en su vida puede ser interrumpida si decidieran abandonar a la empresa

Con relación al compromiso normativo un $26.67 \%$ mencionó que la empresa merece su lealtad, un 53.33\% indicaron retirarse de la empresa si estuvieran presionados. El $40 \%$ indicó estar en deuda con la empresa. Un 33.33\% de los encuestados mencionaron seguir en la empresa por obligación moral. El 33.33\% indicaron no estar ni de acuerdo ni en desacuerdo con respecto a que, si tuvieran otras oportunidades laborales, no sería correcto dejar a la empresa ahora y por último $33.33 \%$ indicó sentirse culpable si abandonara la empresa

De acuerdo al análisis de Pareto, pudimos resaltar tres preguntas críticas. La primera pregunta es el número nueve, perteneciente a la subcategoría compromiso de continuidad, donde el $80 \%$ de los encuestados indicaron que el trabajo no cumple sus expectativas, para seguir perteneciendo, y está relacionado a la falta de motivación. La segunda pregunta crítica es el número tres, pertenece a la subcategoría compromiso afectivo, donde el $66.67 \%$ de los encuestados mencionaron que no sienten suyos los problemas de la organización son por ello que nos damos cuenta de la falta de involucramiento e identificación con la empresa por parte de los asesores de ventas. Y por último resalta la pregunta crítica número trece en la que un $59.99 \%$ de encuestados indicaron no tener lealtad hacia la empresa.

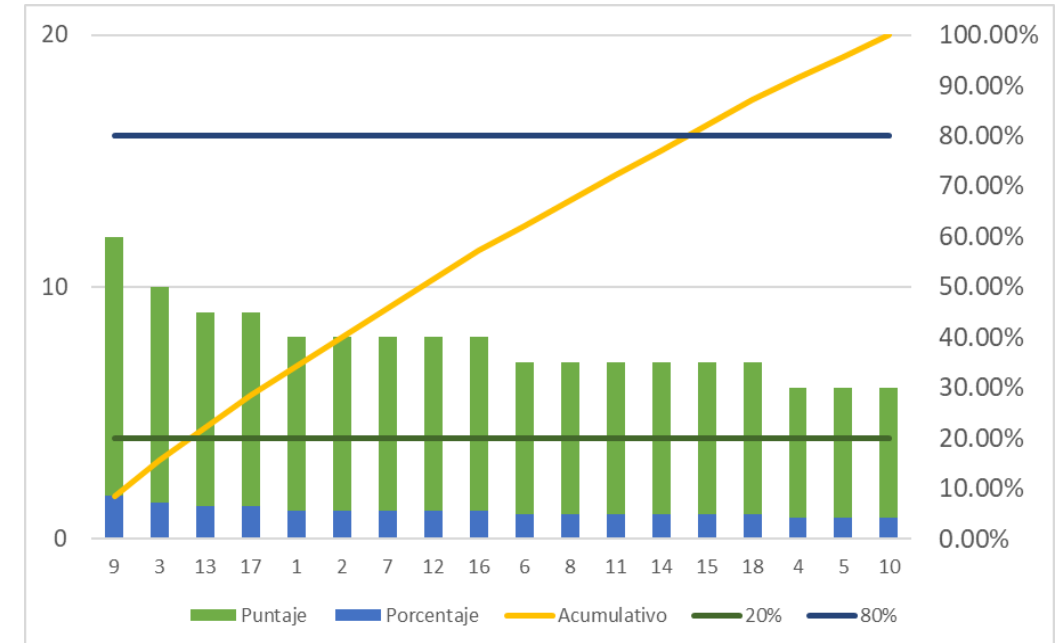

Figura 1. Diagrama de Pareto de la categoría compromiso organizacional 


\section{Resultados cualitativos}

Las entrevistas realizadas a los gerentes fueron respecto: ¿En su opinión como es el compromiso organizacional de los trabajadores de la empresa? ¿que sugiere?: los tres entrevistados indicaron que el compromiso organizacional es bajo debido a que los asesores de ventas están en el trabajo solamente por el valor monetario y no les importa nada más, tratan de ahorrar su dinero para retirarse, además les falta tomar las cosas en enserio ya que descuidan el trabajo teniendo que repetir muchas veces lo que no deben de hacer, algunos asesores están comprometidos con la visión de la empresa, mientras que la mayoría están equivocados; por ejemplo, muchas asesoras de venta piensan que cumplir sólo con el horario de trabajo significa compromiso organizacional; es decir, no demuestran un desempeño óptimo e involucramiento al cien por ciento con la empresa, por más que la entidad les brinda apoyo de muchas formas, no se logra una fidelización con la empresa y muchas de ellas deciden abandonar la empresa, es por ello que se sugirió generar compromiso mediante del involucramiento de la empresa con los colaboradores trabajando el lado emocional, realizando diversas actividades desde charlas, reuniones, bienestar social con la finalidad de crear un involucramiento con la empresa.

La siguiente pregunta es respecto: ¿En su opinión como es el compromiso afectivo (identificación y sentimiento) de los trabajadores de la empresa? ¿Qué sugiere? Mencionaron que afortunadamente la marca de los insumos de son de una distribuidora de productos de belleza tanto facial, capilar y corporal, esto ha contribuido a que las colaboradoras se fidelicen con la marca, generando empatía, aprendiendo sobre tratamientos de belleza, cuidado la piel y otros aspectos. Para este tipo de compromiso se sugirió realizar actividades de integración para que las asesoras trabajen en equipo, se alienten, buscando mejorar los resultados en las ventas.

La siguiente pregunta es relacionada al compromiso de continuidad ¿En su opinión como es el compromiso de continuidad (motivación salarial, estabilidad) de los trabajadores de la empresa? ¿Qué sugiere? Señalaron que los asesores están de una manera están estables por el buen sueldo que mantienen ya que lo que ganan está un poco por encima de la media que maneja la industria de belleza, además se les apoya cuando tienen problemas personales económicos brindándoles préstamos sin ningún interés ni tampoco tramites, se da un monto de monto de dinero por tienda para que compren cosas como agua, gaseosas, galletas, dulces, etc., pero lamentablemente a pesar de todo la estabilidad que se habla solo es de un corto plazo, es decir que abandonan las empresa. Este en este punto se sugirió trabajar emocionante para que las trabajadoras se den cuenta y valoren lo que la empresa les brinda y de esa forma lograr el compromiso organizacional sobre todo porque no ven una relación a largo plazo

Y por último se destacó la pregunta ¿En su opinión como es el compromiso normativo (obligación moral, y lealtad) de los trabajadores de la empresa? ¿Por qué? ¿Qué sugiere? Mencionaron las entrevistadas que de alguna manera los asesores de ventas tienen una obligación moral porque se dan cuenta que muchas veces se les ayudado de cualquier forma, sobre todo económicamente brindándoles prestamos, pero con relación a la lealtad les falta porque hay personas que deciden abandonar la empresa pensando que otras organizaciones van a ser iguales que esta empresa, luego de un tiempo regresan porque se dan cuenta que afuera las empresas son diferentes. En este caso se sugirió que las asesoras de ventas conozcan más sobre la empresa, 
enseñarles a valorar los esfuerzos que se les otorga, a valorar el aprendizaje que obtienen sobre la industria de la belleza, las capacitaciones

Como diagnostico final entre los resultados cuantitativos y cualitativos se pudo determinar los siguientes problemas más relevantes: falta de involucramiento de los trabajadores hacia la organización, además no están cumpliendo sus expectativas en el trabajo, es decir falta de motivación y por ultimo rotación en el trabajo, es por ello que se planteó lo siguiente:

\section{Implantación del modelo IML}

Este modelo IML implica realizar actividades mediante un plan de involucramiento para los asesores de ventas, plan de motivación y la implementación de una línea de carrera para lograr aumentar el compromiso organizacional.

Objetivo1: Incrementar el involucramiento en los asesores de ventas Actividades:

Difusión continua de las políticas, visión, misión y reglamento, mediante exposiciones además se les hará entrega a los colaboradores de trípticos y se publicará en redes sociales sobre las políticas y reglamento, para que estén a la vista y lo pongan en práctica.

$>$ Reforzar los valores, mediante charlas con experto del tema, además se hará entrega en cuadro tanto los valores, misión y visión para ser colgados en cada tienda.

$>$ Fomentar el trabajo en equipo a través de objetivos diarios

$>$ Fortalecer el buen clima laboral a través de talleres.

Objetivo2: Incentivar la motivación en los asesores de ventas Actividades:

> Festividad de fechas importantes, consiste en la celebridad de las fechas importantes como será el día de mujer, ya que la mayoría de los trabadores de ventas son mujeres, el día del trabajador, día de la madre, aniversario de la empresa, cumpleaños de cada integrante.

$>$ Premios y reconocimientos, por el esfuerzo que desempeñan los asesores

$>$ Juegos motivacionales, consiste en realizar juegos con la finalidad de tener trabajadores impulsados y motivado, mejorando la comunicación.

$>$ Envío de mensajes motivacionales vía WhatsApp, consiste en enviar mensajes motivacionales a los trabajadores durante un tiempo con el fin de lograr un asesor feliz en el trabajo.

$>$ Se realizará un control de motivación a través de una pequeña encuesta.

Objetivo3: Retener a los asesores de ventas

Actividades:

Fomentar la línea de carrera en los asesores de venta, se realizará una capacitación por una persona especialista sobre el tema, en el que se describen los requisitos, funciones, y 
beneficios, con un logro parcial de la existencia y conocimiento de la línea de carrera por parte de los asesores de ventas.

$>$ Desarrollo profesional personalizado, consiste en otorgarle un cargo solo para evaluar el desempeño del asesor.

$>$ Monitorear y controlar el desempeño, con la finalidad de verificar el cumplimiento de funciones adecuadamente, será a través de una visita sorpresa a las tiendas de un cliente infiltrado con fin obtener todo tipo información, además se verificará el código de cada trabajador para ver la evolución de las ventas que realizan.

$>$ Comparar el desempeño de los trabajadores, después de monitorear se hará una comparación de los asesores de ventas sobre su desempeño del cargo que se la otorgado.

$>$ Evaluación de desempeño

$>$ Identificar al talento, después de realizar la evaluación se identificará al trabajador adecuado para el nuevo cargo

> Asignar al ganador, esta última actividad consiste en asignar al asesor que cumplió con los requisitos requeridos de todo el procedimiento.

\section{Conclusiones}

Se planteó el modelo IML, con la finalidad de aumentar el compromiso organizacional basándose en hacer un plan para mejorar el involucramiento laboral, plan de incentivar la motivación y la implementación de una línea de cerrera para retener a los trabajadores de ventas.

Para analizar la problemática, se desarrolló una recolección de información tanto cualitativa como cuantitativa en la que se determinó que hay una deficiencia respecto al compromiso organizacional debido a que asesores de ventas no se involucran como empresa, pero si tienen una identificación como marca. Por otro lado, se evidenció que la empresa no motiva emocionalmente ni se involucra mucho con los trabajadores.

Los factores más relevantes que se determinaron fueron la falta de motivación ya que la empresa no está cumpliendo sus expectativas motivacionales, porque en el nivel económico están satisfechas. El otro factor que resaltó la falta de identificación con los problemas de la empresa, aunque les gusta el rubro que manejan, y por último es la falta de lealtad laboral.

\section{Bibliografía}

Alles, M. (2007). comportamiento organizacional:como lograr un cambio cultural a través de gestión por competencias. Buenos Aires: Granica.

Amoros, E. (2007). Comportamineto organizacional . Perú: Biblioteca Virtual Eumednet.

Aon. (2018). Tendencias Globales de compromiso del empleado. Aon empower results. Obtenido de https://www.aon.com/getmedia/ae71f042-e46a-459b-b5fe8034d006a836/ESP_Tendenciales-Globales-de-Compromiso-de-los-Empleados2018.pdf.aspx

Becker, T. (1992). Foci and Bases of Commitment: Are They Distinctions worth Making? The Academy of Management Journal, 35(1), 232-244. Obtenido de https://www.researchgate.net/profile/Thomas_Becker14/publication/246208329_Foci_an d_Bases_of_Commitment_Are_They_Distinctions_Worth_Making/links/5639357108ae4 624b75efdb1/Foci-and-Bases-of-Commitment-Are-They-Distinctions-Worth-Making.pdf 
Cegarra , J. (2012). Los métodos de investigación cientifica y tecnologíca. Madrid: Diaz de Santos. Chiavenato, I. (2014). introduccion a la teoria general de la administracion. mexico: McGrawHill. Crespo, C. (2016). clima y compromiso organizacional en una mediana empresa manufactura de San Luis Potosí S.L.P. Mexico (Maestro en Administración con Énfasis en Negocios): Universidad Autonoma de San Luis de Potosí.

Diario Gestión . (21 de noviembre de 2015). El 87\% de empresas considera que la falta de compromiso laboral es su principal problema. Obtenido de https://gestion.pe/tendencias/management-empleo/87-empresas-considera-faltacompromiso-laboral-principal-problema-105592

Fields, D. (2013). taking the measure of work. Estados Unidos : IAP.

Fuentes, K. (2018). Compromiso organizacional y desempeño laboral en los trabajadores de la Unidad de Gestión Educativa Local 01 - San Juan de Miraflores, 2018. (tesis de maestria) Lima : Universidad Cesar Vallejo .

Hernández, R., Fernández, C., \& Baptista, M. (2010). MetodologÍa de la investigación. Mexico: McGrawHill Educación .

Hurtado, J. (2000). metología de la investigación holística. Caracas: Sygal.

Loza, S. (2016). Clima Laboral y su influencia en el Compromiso Organizacional del personal administrativo de Aymesa S.A. ( tesis de titulación) Quito: Universidad Central de Ecuador.

Mercado, S. (2002). Administración aplicada . México : Limusa.

Meyer, J., \& Allen, N. (1991). A three-component conceptualization of organizational commitment. Human resource management review, 1, 61-89.

Paredes , A. (2017). Compromiso Organizacional en Trabajadores de una empresa de Trade marketing del distrito de San Borja, 2017. (Tesis de licenciatura) Lima: Universidad Inca Garcilaso de la Vega.

Robbins, S., \& Coulter, M. (2014). Admnistración. Mexico : Pearson.

Romeu, E. (1999). Aplicación del enfoque comunicativo en la escuela media. La Habana: Pueblo y Educación. 